\section{Taking ABAT to OA}

New research establishes the enzyme 4-aminobutyrate aminotransferase, mitochondrial (ABAT) as a key regulator of cellular metabolism and a potential therapeutic target in osteoarthritis (OA). The research also demonstrates that vigabatrin, a small-molecule inhibitor of ABAT that is already approved for clinical use as an anticonvulsant, prevents the development of $\mathrm{OA}$ in a mouse model of the disease.

Previous studies by the same group had revealed that genetic knockdown of the gene encoding DNA methyltransferase $3 \mathrm{~B}$
(DNMT3B) in articular chondrocytes led to OA progression via changes in mitochondrial metabolism. In the current study, the group identified ABAT as an important downstream target of DNMT3B in chondrocytes and elucidated its role in regulating chondrocyte mitochondrial function and the development of OA.

"We found that loss of function of DNMT3B or gain of function of ABAT both lead to increased oxidative phosphorylation and a catabolic chondrocyte phenotype," explains corresponding author Regis O’Keefe. "By contrast, gain of function of DNMT3B or loss of function of ABAT both reduce oxidative phosphorylation and result in an anabolic phenotype." intraperitoneal administration of vigabatrin ... completely prevented the development of injuryinduced OA
Consistent with these findings, lentivirus-mediated overexpression of ABAT in mouse knees accelerated OA development following medial ligament injury (MLI) surgery, whereas lentiviral suppression of ABAT partially attenuated cartilage destruction. Notably, intraperitoneal administration of vigabatrin at a dose of $200 \mathrm{mg} / \mathrm{kg}$ for 6 weeks following MLI surgery completely prevented the development of injury-induced OA. In primary articular chondrocyte cell cultures, vigabatrin treatment also reduced mitochondrial respiration and inhibited IL- $1 \beta$-mediated expression of catabolic genes including Runx2, Mmp13 and Col10a1.

The investigators envisage that ABAT inhibition could be developed as a targeted therapy for $\mathrm{OA}$, and are exploring the possibility of delivering ABAT small interfering RNA into arthritic joints via nanoparticles.

Sarah Onuora

ORIGINAL ARTICLE Shen, J. et al. Inhibition of 4-aminobutyrate aminotransferase protects against injury-induced osteoarthritis in mice. JCl Insight 4. e128568 (2019)

\title{
SYSTEMIC LUPUS ERYTHEMATOSUS
}

\section{Targeting mitochondrial dysfunction in SLE}

Emerging evidence suggests that mitochondrial abnormalities contribute to immune dysregulation and organ damage in systemic lupus erythematosus (SLE). In a new study, targeting mitochondrial dysfunction using the drug idebenone improved clinical and immunological features of lupus-like disease in mice, highlighting idebenone as a promising new drug for SLE.

Idebenone is a coenzyme Q10 synthetic quinone analogue that has antioxidant properties. Notably, this drug is already approved in some countries for the treatment of other conditions associated with mitochondrial dysfunction (such as Duchenne muscular dystrophy).

"In two mouse models of SLE, we found that administration of idebenone reduced renal inflammation and renal function, attenuated systemic immune dysregulation in the innate and adaptive immune systems and improved mitochondrial metabolism," states Mariana Kaplan, corresponding author on the study.

Treatment with idebenone improved the survival of the mice and was well tolerated. "Importantly, the use of idebenone was associated with improvements in endotheliumdependent vasorelaxation, suggesting that this drug could target lupus vasculopathy, a prominent feature in this disease," explains Kaplan.

One mechanism by which mitochondrial dysfunction and aberrant production of mitochondrial reactive oxygen species (mROS) might contribute to SLE is by promoting the formation of neutrophil extracellular traps (NETs) and activation of the type I interferon pathway. Indeed, in neutrophils from mice with lupus or from patients with SLE, ex vivo treatment with idebenone inhibited spontaneous NET formation.

Furthermore, in the mouse neutrophils, this treatment was associated with decreased mROS synthesis.

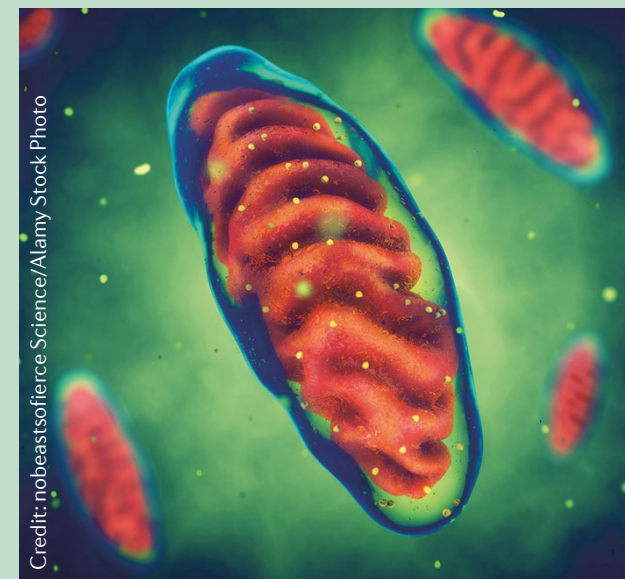

"We are continuing to investigate potential therapeutic targets that modify mitochondrial function and immunometabolism in SLE," says Kaplan. "Our hope is to be able to identify the best compounds that could be tested in clinical trials in SLE in the future." Jessica McHugh

ORIGINAL ARTICLE Blanco, L. P. et al.

The coenzyme $\mathrm{Q} 10$ analog idebenone attenuates murine lupus by improving mitochondrial metabolism and reducing inflammation. Arthritis Rheumatol. https://doi.org/10.1002/ art.41128 (2019) 